\title{
Network construction of aberrantly expressed miRNAs and their target mRNAs in ventricular myocardium with ischemia-reperfusion arrhythmias
}

Jian Tang

Guizhou medical university https://orcid.org/0000-0002-2846-1912

Hong Gao (D2169617@qq.com )

https://orcid.org/0000-0002-3555-5570

Yanqiu Liu

Guizhou medical university

Jing Song

Guizhou provincial tumor hospital

\section{Yurong Feng}

Guizhou medical university

Guilong Wang

Guizhou medical university

Youqin He

Guizhou medical university

Research article

Keywords: reperfusion arrhythmias, microRNAs, mRNA, bioinformatics

Posted Date: July 8th, 2019

DOI: https://doi.org/10.21203/rs.2.11079/v1

License: (c) (1) This work is licensed under a Creative Commons Attribution 4.0 International License. Read Full License

Version of Record: A version of this preprint was published at Journal of Cardiothoracic Surgery on August 12th, 2020. See the published version at https://doi.org/10.1186/s13019-020-01262-4. 


\section{Abstract}

Background: Increasing evidences have verified that microRNAs (miRNAs) play an important role in formation and progression of various cardiac diseases including arrhythmias. Existing research has showed that certain miRNAs exhibit significantly different expressions and effects in arrhythmias. However, the effect of miRNAs in the progression of hypothermic ischemic-reperfusion arrhythmias (RA) and its potential mechanism remains to be further discussed. Methods: By utilizing a model for hypothermic ischemia-reperfusion of rats, miRNAs expression profiles of ventricular myocardium with global hypothermic ischemia-reperfusion and those of ventricular myocardium with hypothermic ischemia-RA were established through high-throughput sequencing. Furthermore, the aberrantly expressed miRNAs in myocardium with and without hypothermic ischemia-RA were screened and verified. By applying RNAhybrid, MiRanda, and TargetScan software, the target genes of these aberrantly expressed miRNAs were predicted. Based on Gene Ontology (GO) and Kyoto Encyclopedia of Genes and Genomes (KEGG) databases, the mRNA targets associated with these miRNAs were determined and the miRNA-mRNA interaction during the progression of cardiovascular diseases was explored. The aberrantly expressed miRNAs related to hypothermic ischemia-RA were validated by employing quantitative fluorescence polymerase chain reaction (PCR). Results: Eight significantly aberrantly

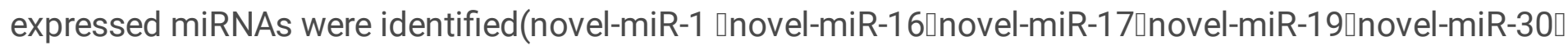
novel-miR-43】rno-miR-122-5p $\llbracket$ rno-miR-429), in which six were up-regulated and two were down-regulated. Moreover, target genes and signaling pathways associated with these aberrantly expressed miRNAs were predicted and analyzed. According to miRNA-mRNA interaction network graph, it was revealed that GJA1 gene was considered as the target of novel-miR-17. Conclusions: Aberrantly expressed miRNAs were possibly associated with the formation mechanism of hypothermic ischemia-RA. Specific miRNAs, such as novel-miR-17 and rno-miR-429 are probably new potential targets for further conducting functional study on hypothermic ischemia-RA.

\section{Background}

Reperfusion arrhythmia (RA) refers to arrhythmia induced by recovering myocardial perfusion after occurrence of coronary occlusion or blocking of myocardial blood flows. It is one of characteristics of myocardial ischemia-reperfusion injury (MIRI). It mainly appears as various ventricular arrhythmias including ventricular premature beat, ventricular tachycardia (VT), and ventricular fibrillation. RA can even trigger hemodynamic disorder to cause sudden cardiac death (SCD) [1-3]. In recent years, with the development of various technologies such as anesthesia and cardiopulmonary bypass (CPB), the effect of cardiac surgical procedures has been greatly improved. However, RA is still the major complication during heart resuscitation through open heart surgery under cardiopulmonary bypass (CPB), which directly influences whether the surgery can be successfully conducted or not and patients' prognosis. Therefore, investigating the formation mechanism of RA after hypothermic ischemia exerts significance on preventing this kind of complications and also provides new targets and directions for clinical treatment. 
MicroRNAs (miRNAs) play a crucial role in pathogenesis and progression of various cardiac diseases. They can lead to the development of certain cardiac diseases by regulating associated target genes [4]. Multiple miRNAs participate in the reconstruction of electrophysiology and ion channel by regulating gene expressions of cardiomyocyte during arrhythmias [5]. Clinical research showed that miRNA-1 in patients' serum greatly rises after conducting CPB [6]. Moreover, Bostjancic et al. [7] validated that two target genes (GJA1 and KCNJ2) are speculated from the nucleotide sequence at 5 ' end of miRNA-1, which are coded as $\mathrm{Cx} 43$ and potassium channel subunits Kir2.1, respectively. The over-expression of mi-RNA-1 can inhibit the expressions of GJA1 and KCNJ2, resulting in the electrolyte disorder of cardiomyocyte to further trigger arrhythmias. In hypothermic ischemia-RA, whether there are miRNAs affecting RA by regulating GJA1 and KCNJ2 or not has not been known.

Gap junction (GJ), as the basic cardiac electrophysiological structure, can sustain normal coupling in electrocardiogram (ECG) signals and mechanical coupling by mediating intercellular electrochemical communication. Connexin (Cx) is the basic unit of GJ [8], and its normal expression is crucial for guaranteeing normal electric coupling and conduction between cardiomyocytes. $\mathrm{Cx} 43$ is subjected to lateralization and dephosphorylation during ischemia-reperfusion injury, thus leading to lateral conduction, formation of reentry and electrical uncoupling to further trigger RA $[9,10]$. By observing myocardium with ischemia-RA, it can be also found that the expression, distribution and phosphatization states of $\mathrm{Cx} 43$ are all changed at different degrees to slow down conduction velocity of myocardium and increase the formation probability of RA [11]. However, the specific correlation between Cx43 and miRNAs in hypothermic ischemia-RA has not been clarified.

Therefore, miRNAs related to hypothermic ischemia-RA were screened by utilizing high-throughput sequencing. By applying bioinformatics analysis methods including GO and KEGG databases, the miRNA-mRNA interaction was further analyzed. On this basis, the potential functions of aberrantly expressed miRNAs and the possible mechanism of interaction between the miRNAs and hypothermic ischemia-RA through genes were determined. It is expected to provide new targets and directions for preventing hypothermic ischemia-RA.

\section{Methods}

Ethical approval The study conformed to the Guide for the Care and Use of Laboratory Animals published by the US National Institutes of Health (NIH Publication No. 85-23, revised 1996). All protocols were approved by the Ethics Committee of Guizhou Medical University(No.1800451).

Preparation of models of isolated rat hearts. Sixteen healthy male SD rats of 2 3 months old weighing 300 400 $\mathrm{g}$ at clean grade were provided by the Experimental Animal Center, Guizhou Medical University (Guiyang, Guizhou Province, China). Heparin (3\%, batch number: 51606118, Jiangsu Wanbang Biochemical Medicine Co., Ltd.) of $500 \mathrm{lu} / \mathrm{kg}$ was intraperitoneally injected for anticoagulation. After administration for $10 \mathrm{~min}, 300 \mathrm{mg} / \mathrm{kg}$ of $10 \%$ chloral hydrate (batch number: 20180429 , Tianjin Kermel Chemical Reagent Co., Ltd.) was intraperitoneally injected for anesthesia. The chest of the rats was 
opened fast after the anesthesia took effects to take the heart, which was then placed in the KrebsHenseleit $(\mathrm{K}-\mathrm{H})$ solution at $4{ }^{\circ} \mathrm{C}$ immediately to trim and expose the aorta. The aorta was fixed on the Langendroff perfusion equipment (Shanghai Alcott Biotech Co., Ltd.) to conduct acyclic retrograde perfusion at constant temperature $\left(37^{\circ} \mathrm{C}\right)$ and constant pressure $(8.65 \mathrm{kPa})$ with the $\mathrm{K}-\mathrm{H}$ solution saturated with $95 \% 02$ and $5 \% \mathrm{CO} 2$. The model for Langendroff perfusion of isolated rat hearts was regarded successfully prepared if the heart rhythm (HR) was recovered within 3 min after balance perfusion and HR was higher than 180 times/min at the end of the balance perfusion.

Experimental treatments. Sixteen prepared models for Langendroff perfusion of isolated rat hearts were divided randomly into two groups ( $\mathrm{n}=8$ in each group): a control group (C group) and an ischemia reperfusion (IR) group. The former was subjected to perfusion continuously for 120 min with $\mathrm{K}-\mathrm{H}$ solution at $37^{\circ} \mathrm{C}$. As for the latter, after balance perfusion with $\mathrm{K}-\mathrm{H}$ solution at $37^{\circ} \mathrm{C}$ for $30 \mathrm{~min}$, Thomas solution of $20 \mathrm{ml} / \mathrm{kg}$ at $4{ }^{\circ} \mathrm{C}$ was injected at the root of the aorta to allow $60 \mathrm{~min}$ of cardiac arrest. The periphery of the heart was protected with $\mathrm{K}-\mathrm{H}$ solution at $4{ }^{\circ} \mathrm{C}$. Then, Thomas solution of $10 \mathrm{ml} / \mathrm{kg}$ at 4 ${ }^{\circ} \mathrm{C}$ was perfused again after cardiac arrest lasted for $30 \mathrm{~min}$ and $\mathrm{K}-\mathrm{H}$ solution at $37^{\circ} \mathrm{C}$ was re-perfused for 30 min after $60 \mathrm{~min}$ of cardiac arrest.

Experimental grouping. The arrhythmias during reperfusion were observed and then quantified according to the Curtis and Walker as well as Lepran grading systems. Rat hearts with scores higher or not higher than 3 were graded to have high risk of IR $(I R-H)$ or low risk of IR (IR-L). Then, the hearts were divided into three groups based on the arrhythmia degree: a C group, an IR-L group, and an IR-H group, each containing specimens of ventricular myocardium of four rats. Myocardium of ventriculus sinister was removed immediately after the perfusion and the hearts were frozen and transferred to a refrigerator to be stored at $-80^{\circ} \mathrm{C}$.

High-throughput sequencing and analysis of aberrant expression of miRNAs. The Trizol method was used to extract the total RNA of the specimens and the RNA was treated with Dnase I to eliminate the DNA pollution. All these procedures strictly followed the instructions. Through the use of the micronucleic acid protein tester (Agilent Company), formaldehyde denaturing gradient gel electrophoresis (DGGE), and capillary electrophoresis, the extracted total RNA was tested to ensure that its concentration and completeness reached the requirements for sequencing. The Beijing Genomics Institute (BGI) was entrusted to perform high-throughput sequencing for the above samples on the BGISEQ-500 platform. The expression of miRNA of the samples was standardized as transcripts per million (TPM). For given transcripts, the gene expression was estimated by aligning the number of fragments in a gene region. The edger software was used to analyze changes in the expression of the gene transcripts of the three groups of samples. After obtaining the value of $P$, multiple hypothesis testing was conducted and the corrected $P$ was represented by false discovery rate (FDR), satisfying FDR $<0.05$. If the fold change in expression was equal to or larger than 2 , the gene was deemed as aberrantly expressed.

Prediction of target genes of aberrantly expressed miRNAs. RNAhybrid, miRanda, and TargetScan [12] databases were used to predict the target genes of aberrantly expressed miRNAs and the predicted ones 
were mapped to each terms in the gene ontology (GO) database to count the number of genes that mapped to each term. Then, hypergeometric distribution was applied to compute the value of $P$. If $P \leq 0.05$, a GO term was regarded to have significantly enriched target genes of aberrantly expressed miRNAs. Then, by using Kyoto Encyclopedia of Genes and Genomes (KEGG) database, target genes of aberrantly expressed miRNAs were mapped and the same calculation method was used to obtain the analysis results of KEGG. Finally, the mRNA-miRNA interaction network graph was preliminarily established.

Analysis of genes associated with the progression of cardiovascular diseases. All of the target mRNAs of differently regulated miRNAs were screened using the TargetScan 7.1. Then, the mRNA-miRNA process was comprehensively analyzed to build networks of the physiological system and pathophysiology. GO and KEGG cardiac function databases were taken to select the mRNAs that participated in pathophysiological process of cardiovascular diseases (CVDs), including hypertrophy, fibrosis, conduction abnormality, and arrhythmia.

Screening of the target miRNAs. Based on gene function analysis, KEGG pathway analysis, and mRNAmiRNA interaction, the bioinformatics analysis was carried out on transcripts of miRNAs related to $\mathrm{KCNJ} 2 / \mathrm{GIA} 1$ genes. The target miRNAs regulating expressions of KCNJ2 or GJA1 in the hypothermia ischemic-RA were preliminarily screened.

Fluorogenic quantitative PCR validation. The Trizol method was used to dissociate and extract the RNA. The ultraviolet spectrophotometer was used to detect the purity of total RNA and for quantification. After subpackage, the RNA was preserved at $-80^{\circ} \mathrm{C}$. The reverse transcription was performed according to the instructions of ThermoScriptTM RT-PCR System (Invitrogen, the United States) and the obtained cDNA was preserved at $-20^{\circ} \mathrm{C}$ for later use. The primer was synthesized by Wuhan Biofavor Biotechnology Services Co., Ltd. The SYBR Premix Ex TaqTM reagent was used and $20 \mu$ l of reaction system contained cDNA $(2 \mu \mathrm{l}), 2 \times$ Master Mix (10 $\mu \mathrm{l}), 20 \times$ TaqMan@ probe and primer mixture $(1 \mu \mathrm{l})$, and double distilled water $(7 \mu \mathrm{l})$. The polymerase chain reaction (PCR) was performed under the following conditions: initial denaturation for 5 min at $94^{\circ} \mathrm{C}$, followed by 40 cycles at $94{ }^{\circ} \mathrm{C}, 60^{\circ} \mathrm{C}$, and $72{ }^{\circ} \mathrm{C}$, respectively for $45 \mathrm{~s}$, and finally extending for $7 \mathrm{~min}$ at $72{ }^{\circ} \mathrm{C}$. The fluorogenic quantitative PCR detecting system was used for carrying out PCR amplification and the solubility curves were drawn. The final data were analyzed using the 2- $\triangle \triangle \mathrm{Ct}$ method.

Statistical analysis All data are presented as the means \pm standard errors of the mean. The incidence was compared with Fisher's exact test, and different experimental groups were compared by Student's t test and analysis of variance using SPSS 22.0 (IBM SPSS Statistics, USA). P $<0.05$ was considered to indicate a statistically significant difference among the all groups.

\section{Results}

Aberrant expression of miRNAs. Some 85 significantly aberrantly expressed miRNAs commonly existing in the three groups were predicted using the miRDeep2 software(Fig.1a). Among the miRNAs with 
significant aberrant expressions, expressions of 46 miRNAs were up-regulated in the $C$ and IR-H groups while those of 9 ones were down-regulated(Fig.1b). In the $C$ and IR-L groups, 32 miRNAs were found to have up-regulated expressions while 9 ones showed down-regulated expressions(Fig.1c). As for the IR-H and IR-L groups, 28 miRNAs had up-regulated miRNAs and 20 ones exhibited down-regulated miRNAs(Fig.1d).

\section{Screening of miRNAs associated with hypothermia ischemic-RA. According to the principle of fold} change $\geq 2$ and FDR $<0.05$, 8 miRNAs most closely associated with the hypothermia ischemic-RA were selected from the 85 aberrantly expressed miRNAs (Table 1). A comparison of expressions of the miRNAs revealed that the three groups had discrepancies in the expressions of novel-miR-1 and novel-miR-16, and IR-H and IR-L groups showed difference in expressions of novel-miR-16 and novel-miR-30. The RA groups and the $\mathrm{C}$ control also displayed differences in rno-miR-122-5p expression. A significant increase in the expression of novel-miR-17 was observed in the IR-H group compared with the IR-L and C groups. Although the expressions of novel-miR-1 and novel-miR-16 did not exhibit significant differences in the three groups, they had higher expressions in the IR-H group.

Predicting target genes of aberrantly expressed miRNAs. RNAhybrid and miRanda were used to predict target genes of aberrantly expressed miRNAs. On this basis, it was found that each miRNA had multiple predicted target genes. Totally 2,810 target genes were predicted for the 8 aberrantly expressed miRNAs. These target genes are probably the targets related to hypothermia ischemic-RA.

GO and KEGG data analysis. Three GOs separately described the biological process (BP), molecular function (MF), and cellular component (CC) of genes. In the current research, aberrantly expressed miRNAs were enriched in numerous CCs, included cell junction, proteinaceous extracellular matrix $\llbracket$ cell projection $\square$ dendrite $\square$ basolateral plasma membrane(Fig.2a). Likewise, the molecular function ,delayed rectifier potassium channel activity $\square$ RNA polymerase II core promoter proximal region sequence-specific DNA binding $\square$ protein kinase binding $\square$ chromatin binding.were also influenced(Fig.2b). The biological process under influences included potassium ion transport across cell membranes, delayed rectifier potassium channels, and regulation of heart rate by cardiac conduction(Fig.3a). Then, enrichment of target genes of aberrantly expressed miRNAs in KEGG pathways was further analyzed and the results showed that the target genes took part in 6 signaling pathways correlated with cardiac diseases(Fig.3b). These signaling pathways mainly included those of epinephrine, arrhythmogenic right ventricular cardiomyopathy (ARVC), and cardiac hypertrophy.

Bioinformatics data. Totally 151 mRNAs showed miRNA-mRNA interactions (Fig. 4). We observed the correlations of these miRNAs with various cardiac pathophysiological processes and the process that they were likely to trigger RA or finally developed to RA (Fig.5). The relationship between the two analyses displayed that 19 of the 151 mRNAs were associated with the development of cardiac diseases (Fig. 6). The target gene GJA1 of novel-miR-17 participated in the proarrhythmia and the associated pathways, which is consistent with previous research results. The target gene KCNQ4 of rno-miR-429 and novel-miR17 was associated with the delayed rectifier potassium channel and the potassium ion transport across 
cell membranes. Through screening using the pathway analysis software, no target gene associated with the electrocardiological mechanism regulated by novel-miR-1 nnovel-miR-13 and novel-miR-43 was found.

RT-qPCR validation of aberrantly expressed miRNAs. Four miRNAs that were closely associated with the RA formation mechanism were selected from the 8 miRNAs and then validated through the use of qRTPCR. It was found that these four miRNAs were novel-miR-17, novel-miR-30, rno-miR-122-5p, and rno-miR429. The results revealed that the change trend of expressions of the four miRNAs agreed with the results of high-throughput sequencing (Fig.7).

\section{Discussion}

The miRNAs regulate the gene expression by inhibiting the translation process of protein-coding mRNAs through combination with the 3' untranslated region (3'UTR) of specific mRNAs [13-15]. Research in recent years has revealed that miRNA molecules played a significant role in the progression of cardiac diseases such as MIRI and arrhythmia $[16,17]$. Human intervention on the expression of some miRNAs affects the progression of RA, the formation mechanism of which is closely associated with the electrophysiological reconstruction. The miRNAs are key molecules in arrhythmia, so their intervention and regulation has become a new target for treatment of diseases $[18,19]$. The target genes of the four miRNAs newly discovered in the research are mainly found in the ARVC pathway, while effects of these miRNAs in biological and pathological research have not been reported yet. Therefore, clarifying whether these miRNAs have important influences on the RA by regulating relevant signaling pathways provides more possible directions for the research on hypothermia ischemic-RA.

Aberrantly expressed miRNAs and their target protein-coding RNAs consist of a complicated network, which participates in the regulation of the formation and progression of RA. Previous research has reported that miRNAs and Cx43 take part in the formation of RA [20], while the correlation mechanism between the two is rarely investigated in detail. Apart from ventricular arrhythmia, upward shift of the S-T segment, and QT prolongation, the ECG also exhibited prolonged QRS interval after ischemia reperfusion, all of which are associated with a slower intermyocardial conduction velocity after ischemia reperfusion [21]. GJ, as the basic structure of cardiac electrophysiology, maintains the normal coupling in EGC signals and mechanical coupling by mediating intercellular electrochemical communications. The normal expression of $\mathrm{Cx} 43$ that is coded by gene GJA1 is crucial for the electric coupling and conduction between cardiomyocytes [22]. We also found that the expression, distribution, and phosphorylation state of $\mathrm{Cx} 43$ in the myocardium of ischemic-reperfusion arrhythmia all changed at different degrees, thus slowing down the conduction velocity of myocardium and increasing the formation of RA [23, 24]. Therefore, regulating the expression of $\mathrm{Cx} 43$ is one of the key factors for the formation of hypothermia ischemic-RA. In the research, GJA1 was the target of novel-miR-17. Whether novel-miR-17 can regulate $\mathrm{C} \times 43$ protein by acting on CJA1 and therefore influence the formation of hypothermia ischemic-RA will be a future research topic. 
Through GO functional and KEGG pathway enrichment analyses, it was found that target genes of the eight significantly aberrantly expressed miRNAs took part in the potassium ion transport across cell membranes and the delayed rectifier potassium channels. Therefore, it can be assumed that aberrantly expressed miRNAs are capable of regulating the target genes to participate in the action mechanism of hypothermia ischemic-RA via the above channels. After suffering hypothermic ischemic injury, the electrophysiological changes in irregular myocardium repolarization including MAPD prolongation and increasing transmitted drug resistance (TDR) in the myocardium indicated that the potassium channel possibly took part in the formation of RA $[25,26]$. According to the regulation network of the aberrantly expressed miRNAs and their mRNAs, it was revealed that the relevant target genes KCNQ4 and KCNA6 of rno-miR-429 and novel-miR-17 were deemed associated with the potassium ion transport across cell membranes and delayed rectifier potassium channels. Therefore, they can lead to the formation of RA by participating in the regulation of the ion channels, and the specific regulation mechanism will be an important topic in future research. Moreover, the miRNA-mRNA interaction also indicated that seven mRNAs (Cacna1c, Itgb3, Ctnnb1, Gja1, Itgav, Cacnb3, and Tcf7I1) are probably the targets of the candidate miRNAs that take part in the progression of CVDs. This provides more possible targets and directions for the future research.

\section{Conclusion}

In conclusion, Novel-miR-17 probably has effects on the CJA1 gene, thus regulating the formation of RA. Meanwhile, the biological analysis reveals that novel-miR-17 and rno-miR-429 probably regulate their target genes to influence the ion channels and affect the formation of RA. This indicates that novel-miR17 is possibly a potential biological marker. We will further study the functions of these miRNAs, so as to broaden our knowledge on the mechanism of action of hypothermia ischemic-RA.

\section{Abbreviations}

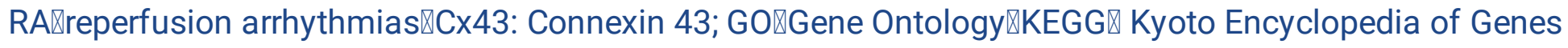

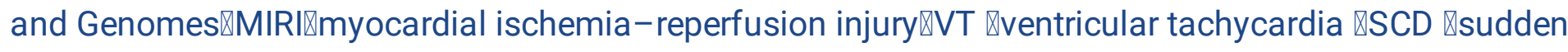

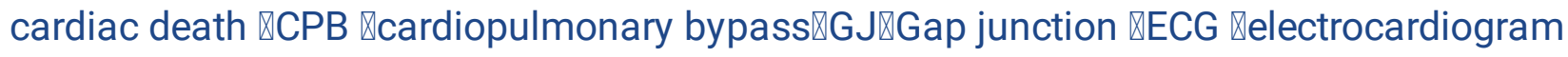

\section{Declarations}

\section{Ethics approval and consent to participate}

The study conformed to the Guide for the Care and Use of Laboratory Animals published by the US National Institutes of Health (NIH Publication No. 85-23, revised 1996). All protocols were approved by the Ethics Committee of Guizhou Medical University (No. 1800451).

\section{Consent for publication}

Not applicable.

\section{Availability of data and materials}


All raw data is available upon request and the corresponding author, Prof. Hong Gao (E-mail: 2169617@qq.com) should be contacted if someone wants to request the data.

\section{Competing interests}

The authors declare no potential conflicts of interest. The authors alone are responsible for the content and writing of the paper.

\section{Funding}

No funding was received.

\section{Authors' contributions}

HG designed the research/study. JT performed the research/study. FYR and WGL collected the data. JS $\square$ HYQ and LYQ analysed the data. All authors read and approved the final manuscript.

\section{Acknowledgements}

Not Applicable.

\section{Reference}

[1] Bernikova OG, Sedova KA, Arteyeva NV, Ovechkin AO, Kharin SN, Shmakov DN, Azarov JE. Repolarization in perfused myocardium predicts reperfusion ventricular tachyarrhythmias[J]. Journal of electrocardiology,2017. [2] Patil K D, Halperin H R, Becker L B. Cardiac arrest: resuscitation and reperfusion[J]. Circulation research,2015,116(12):2041-2049. [3] Gonca E, Darici F. The effect of cannabidiol on ischemia/reperfusion-induced ventricular arrhythmias: the role of adenosine A1 receptors[J]. Journal of cardiovascular pharmacology and therapeutics,2015,20(1):76-83. [4] Ananília Medeiros Gomes da Silva,Jéssica Nayara Góes de Araújo,Katiene Macêdo de Oliveira,et al. Circulating miRNAs in acute new-onset atrial fibrillation and their target mRNA network[J]. Cardiovasc Electrophysiol.2018,29(8):1159-1166.

[5] Wehling M. Translational medicine : science or wishful thinking[J] Transl Med $₫ 2008,6: 31$. [6] Zhou X, Mao A, Wang X, Duan X, Yao Y, Zhang C. Urine and serum microRNA-1 as novel biomarkers for myocardial injury in open-heart surgeries with cardiopulmonary bypass[J]. Plos one,2013,8(4):e62245. [7] Bostjancic E, Zidar N, Stajer D, Glavac D. MicroRNAs miR-1, miR-133a, miR-133b and miR-208 are dysregulated in human myocardial infarction[J]. Cardiology,2010,115(3):163-169. [8] Elnakish MT, Canan BD, Kilic A, Mohler PJ, Janssen PM. Effects of zacopride, a moderate IK1 channel agonist, on triggered arrhythmia and contractility in human ventricular myocardium[J]. Pharmacological research,2017,115:309-318. [9] Veeraraghavan R, Lin J, Keener JP, Gourdie R, Poelzing S. Potassium channels in the $\mathrm{Cx} 43$ gap junction perinexus modulate ephaptic coupling: an experimental and modeling study[J] .Pflugers Arch., 2016, 468ه10®: 1651-61. [10] Desplantez T. Cardiac Cx43, Cx40 and Cx45 coassembling: involvement of connexins epitopes in formation of hemichannels and Gap junction 
channels[J]. BMC cell biology,2017,18(Suppl 1):3. [11] Li WC, Gao H*, Gao J, Wang ZJ. Antiarrhythmic effect of sevoflurane as an additive to HTK solution on reperfusion arrhythmias induced by hypothermia and ischaemia is associated with the phosphorylation of connexin 43 at serine 368[J] .BMC Anesthesiol, 2019, 19(1): 5. [12] Agarwal V, Bell GW, Nam J, Bartel DP. Predicting effective microRNA target sites in mammalian mRNAs. eLife, 4:e05005, (2015)

[13] Suzuki HI, Miyazono K: Emerging complexity of microRNA generation cascades. J Biochem 2011;149:15-25.

[14] Porrello ER: microRNAs in cardiac development and regeneration. Clin Sci (Lond) 2013;125:151-166.

[15] Condorelli G, Latronico MV, Cavarretta E: microRNAs in cardiovascular diseases: current knowledge and

the road ahead. J Am Coll Cardiol 2014;63:2177-2187.

[16]Creemers EE, Tijsen AJ, Pinto YM. Circulating microRNAs: novel biomarkers and extracellular communicators in cardiovascular disease? Circ Res.2012;110(3):483-95.

[17]Mishra PK, Tyagi N, Kumar M, Tyagi SC. MicroRNAs as a therapeutic target for cardiovascular diseases. J Cell Mol Med. 2009;13(4):778-89.

[18]Yang B, Lin $\mathrm{H}$, Xiao J, et al. The muscle-specific microRNA miR-1 regulates cardiac arrhythmogenic potential by targeting GJA1 and KCNJ2.[J]. Nature medicine,2007,13(4):486-491.

[19]Hirt MN, Werner T, Indenbirken D, Alawi M, Demin P, Kunze AC, Stenzig J, Starbatty J, Hansen A, Fiedler $\mathrm{J}$, Thum T, Eschenhagen T: Deciphering the microRNA signature of pathological cardiac hypertrophy by engineered heart tissue- and sequencing-technology. J Mol Cell Cardiol 2015;81:1-9.

[20]Nagomi Sato $\otimes$ Pathological remodeling of cardiac gap junction connexin 43-With special reference to arrhythmogenesis [J]هPathophysiology,2010,17(2):73-81.

[21] Gonca E, Darici F. The effect of cannabidiol on ischemia/reperfusion-induced ventricular arrhythmias: the role of adenosine $\mathrm{A} 1$ receptors[J]. Journal of cardiovascular pharmacology and therapeutics,2015,20(1):76-83. [22] Li J, Goossens S, van Hengel J, Gao E, Cheng L, Tyberghein K, Shang $X$, De Rycke R, van Roy F. Loss of alphaT-catenin alters the hybrid adhering junctions in the heart and leads to dilated cardiomyopathy and ventricular arrhythmia following acute ischemia[J] . Journal of cell science,2012,125(Pt 4):1058-1067. [23] Veeraraghavan R, Lin J, Keener JP, Gourdie R, Poelzing S. Potassium channels in the $\mathrm{Cx} 43$ gap junction perinexus modulate ephaptic coupling: an experimental and modeling study[J] .Pflugers Arch., 2016, 468囚10囚: 1651-61. [24] Desplantez T. Cardiac Cx43, Cx40 and $\mathrm{Cx} 45$ co-assembling: involvement of connexins epitopes in formation of hemichannels and Gap junction channels[J]. BMC cell biology,2017,18(Suppl 1):3. [25] He J, Xu Y, Yang L, Xia G, Deng N, Yang Y, Tian Y, Fu Z, Huang Y. Regulation of inward rectifier potassium current ionic channel remodeling by AT - 
Calcineurin-NFAT signaling pathway in stretch-induced hypertrophic atrial myocytes[J]. Cell Biol Int , 2018, undefined: undefined . [26] Wang LH, Yu CH, Fu Y, Li Q, Sun YQ. Berberine elicits anti-arrhythmic effects via IK1/Kir2.1 in the rat type 2 diabetic myocardial infarction model[J] .Phytother Res, 2011, 25: 33-7.

\section{Table}

Tab. 1 Eight microRNAs with the highest expression among 85 differentially expressed microRNAs

\begin{tabular}{|c|c|c|c|c|}
\hline Mature-ID & Fold change & P value & Regulation & FDR \\
\hline novel-miR-1 & 1.57 & 0.0000045 & up & $<0.001$ \\
\hline novel-miR-16 & 4.32 & 0.0000562 & dpwn & $<0.001$ \\
\hline novel-miR-17 & 2.57 & 0.0000765 & up & $<0.001$ \\
\hline novel-miR-19 & 5.87 & 0.0000694 & up & $<0.001$ \\
\hline novel-miR-30 & 6.63 & 0.0000425 & up & $<0.001$ \\
\hline novel-miR-43 & 7.13 & 0.0000238 & up & $<0.001$ \\
\hline rno-miR-122-5p & 2.38 & 0.0000758 & up & $<0.001$ \\
\hline rno-miR-429 & 5.34 & 0.0000346 & down & $<0.001$ \\
\hline
\end{tabular}

\section{Figures}



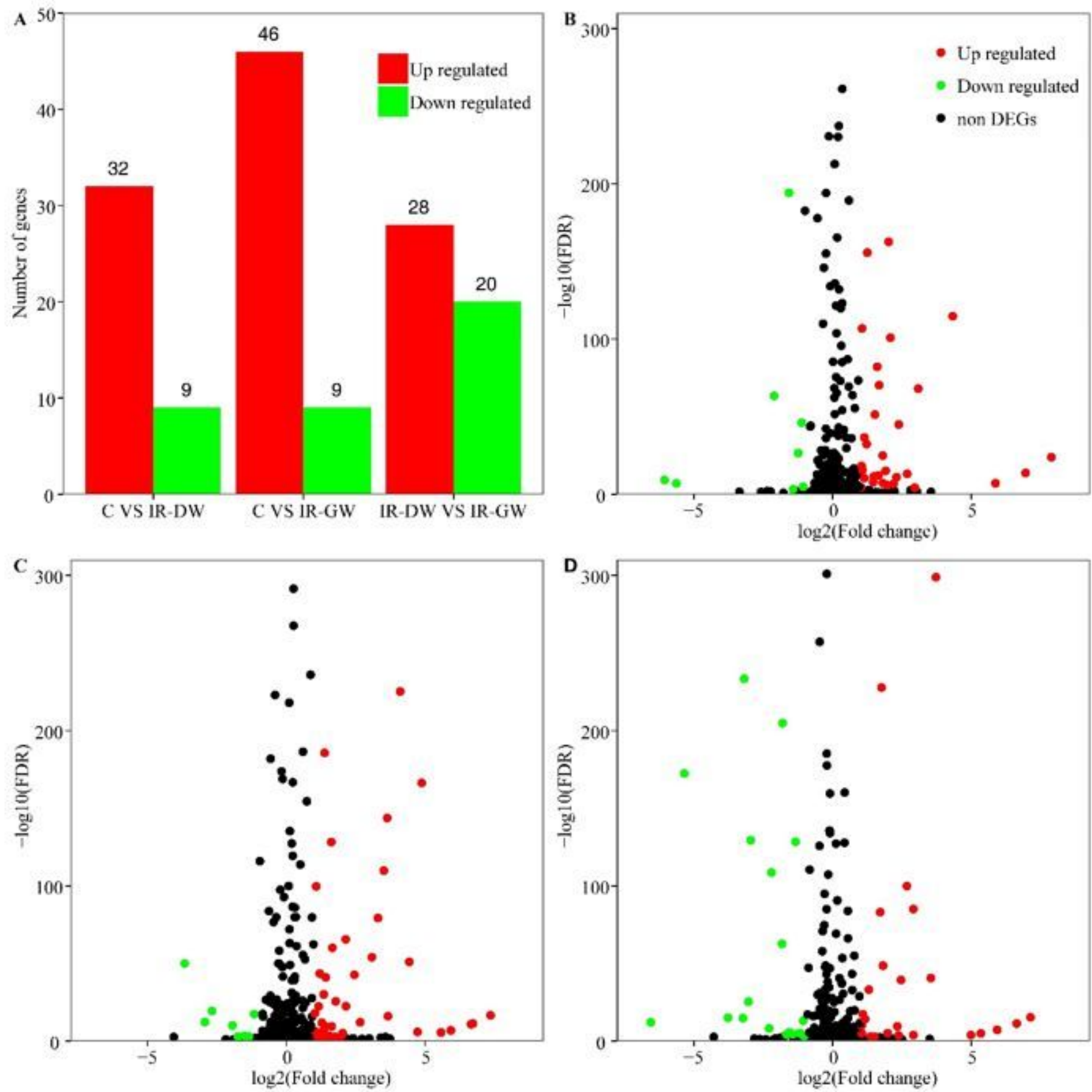

\section{Figure 1}

The plots of differentially expressed microRNAs. A. Group names are listed at the bottom. The number of the significantly $(p<0.1)$ expressed microRNAs are shown on the left. Significant differential expression of 85 microRNAs: green, downregulation; red,upregulation. B.volcano plot of differentially expressed microRNAs from high-throughput sequencing. (C vs IR-DW). The horizontal lines correspond to 2-fold up and down, respectively, and the vertical line represents a FDR. So the red and the green points in the plot represent the differentially expressed microRNAs with significant difference.C. volcano plot of C vs IRGW.D. volcano plot of IR-DW vs IR-GW. 
A

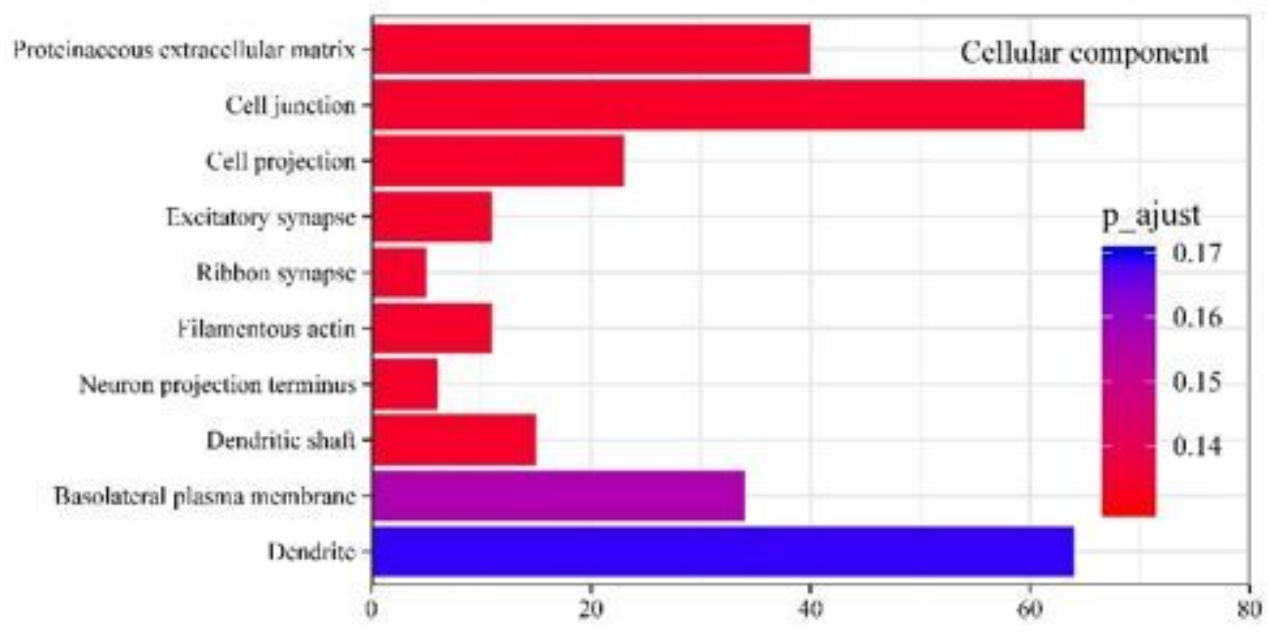

B

RNA polymerase II core promoter proximal region sequence-specific DNA binding Rho guany! nucleotide exchange factor activity

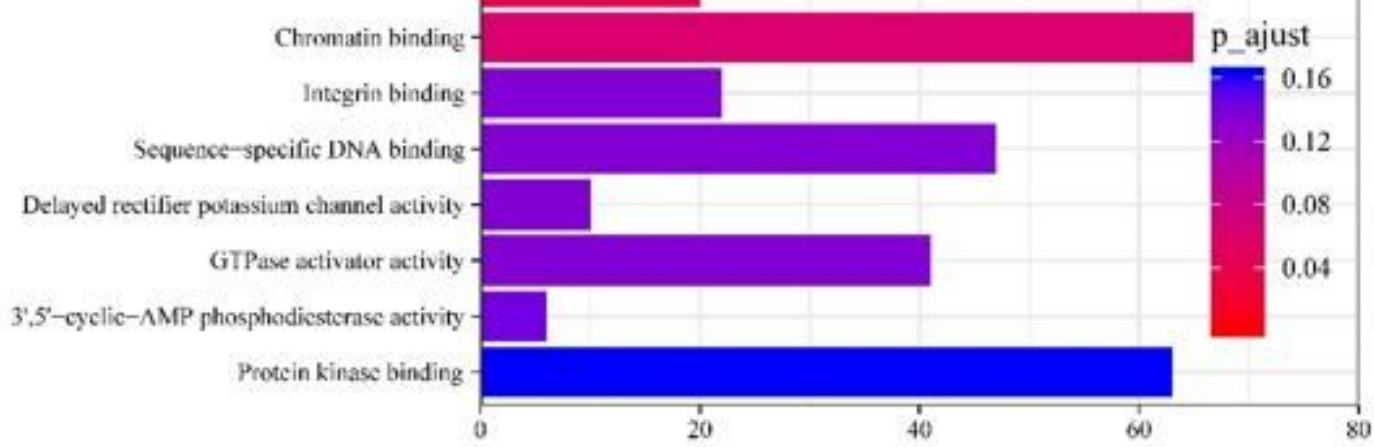

\section{Figure 2}

GO Analysis of differentially expressed MicroRNAs.A.GO analysis for differentially expressed (DE) mRNACellular component (CC). Bar plot explanation(Enrichment Score): the bar plot shows the top ten Enrichment Score value of the significant enrichment terms.B. GO analysis for differentially expressed (DE) mRNA-Molecular function (MF). Bar plot explanation(Enrichment Score): the bar plot shows the top ten Enrichment Score value of the significant enrichment terms. 
A

Rgulation of veniricular eardiac muscle cell membrane repolarization

Regulation of heart rate by cardiac conduction

Regulation of cardiac muscle contraction by regulation of the release of sequestered calcium ion

Regulation of cardiac conduction

Potassium ion transmembrane transport

Positive regulation of cardiac muscle cell proliferation

Negative regulation of cardiac muscle cell proliferation

Negative regulation of cardiac muscle cell apoptotic process

Celaycd rectifier potassium channel activity

Cardiovascular system development

Cirdiac muscle cell diflerentiation

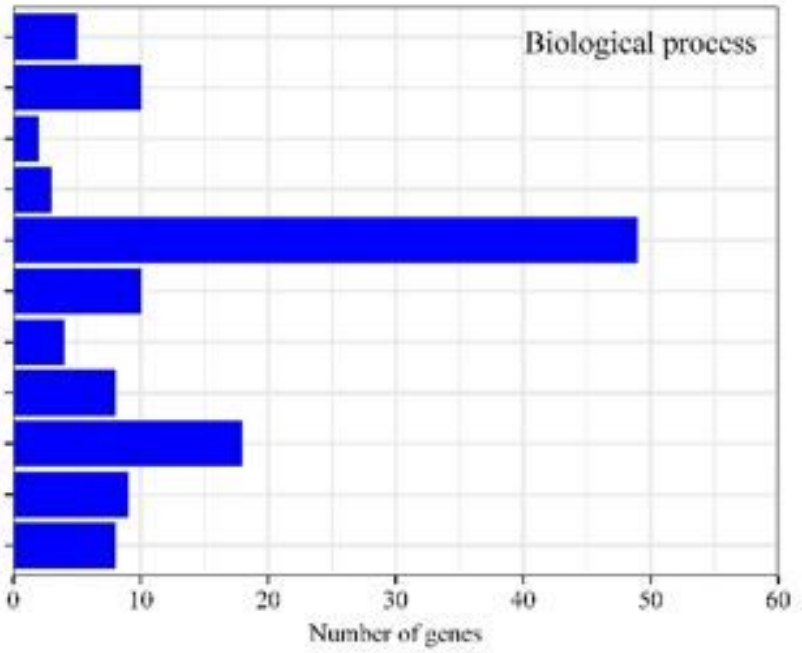

B

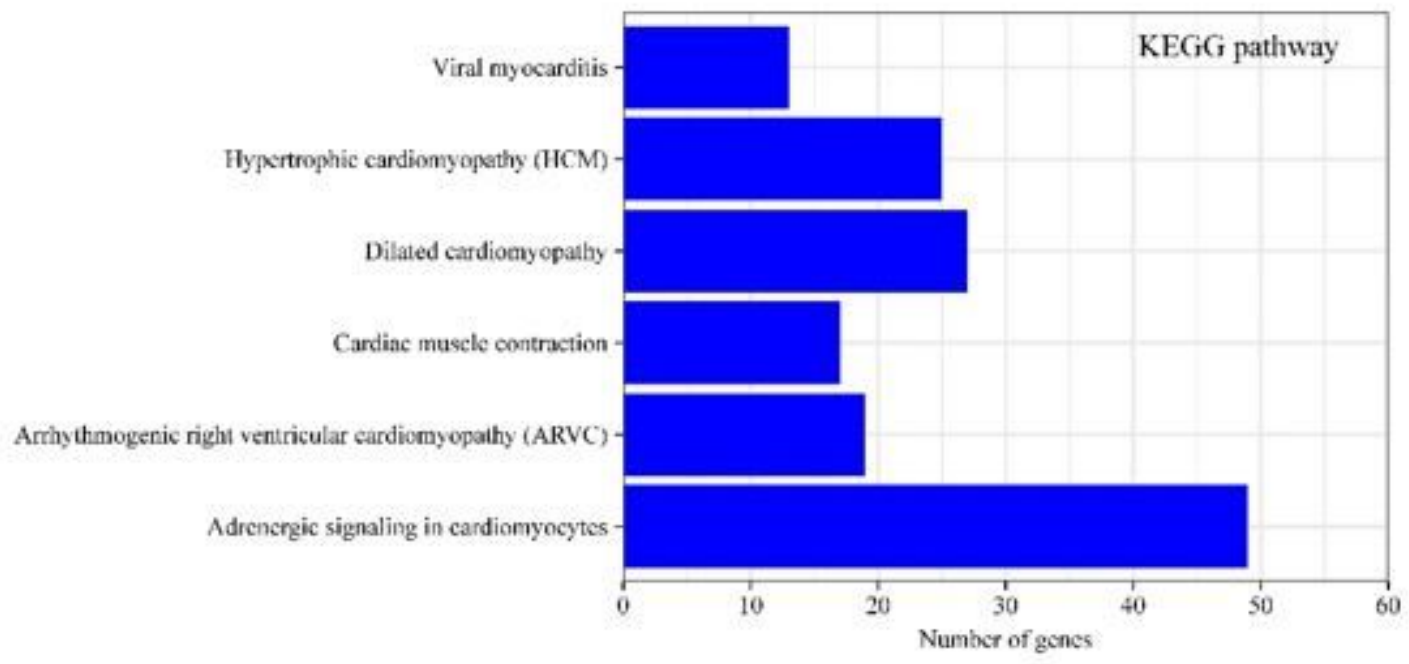

Figure 3

GO Analysis of differentially expressed MicroRNAs.A.GO analysis for differentially expressed (DE) mRNABiological process (BP). Bar plot explanation(Enrichment Score): the bar plot shows the top eleven Enrichment Score value of the significant enrichment terms.B. Pathway analysis of target genes of differentially expressed (DE) microRNAs. Target genes were enriched into different pathways About heart disease based on KEGG pathway database, the top 6 pathways were shown above. 


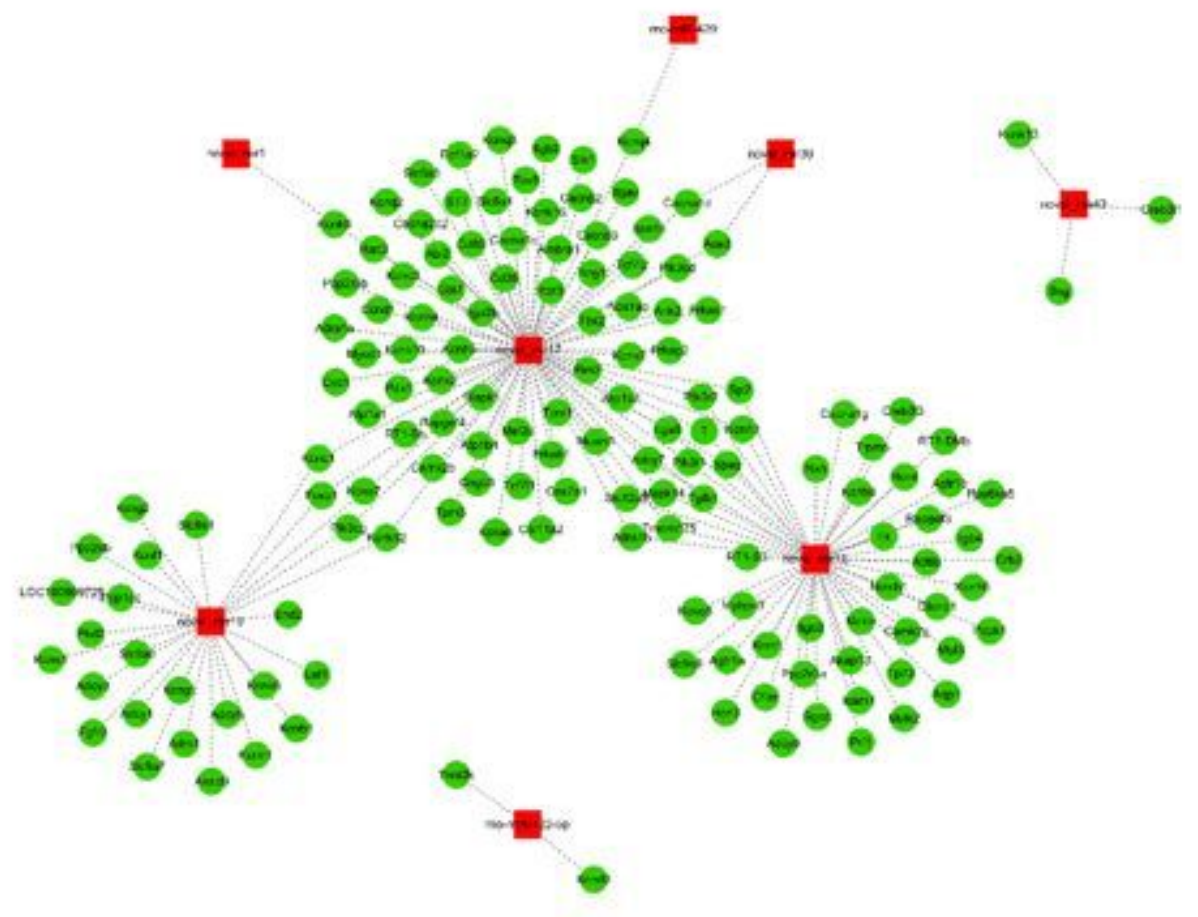

\section{Figure 4}

Interaction of miRNAs with mRNAs involved in cardiovascular processes. It was revealed that GJA1 gene was considered as the target of novel-miR-17. Green box - mRNAs; red symbols - miRNA. cytoscape software

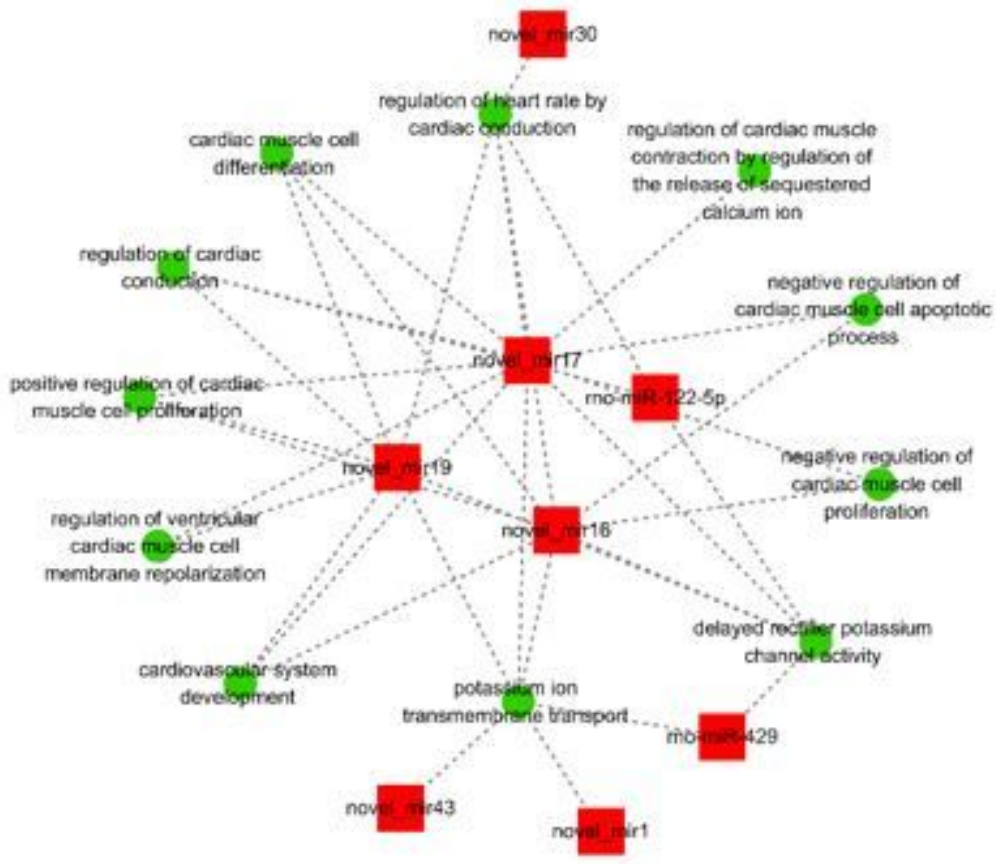

Figure 5 
Interaction of miRNAs with pathophysiological processes related to RA. Green Box - cardiac pathophysiological processes; red symbols - miRNAs. cytoscape software data.

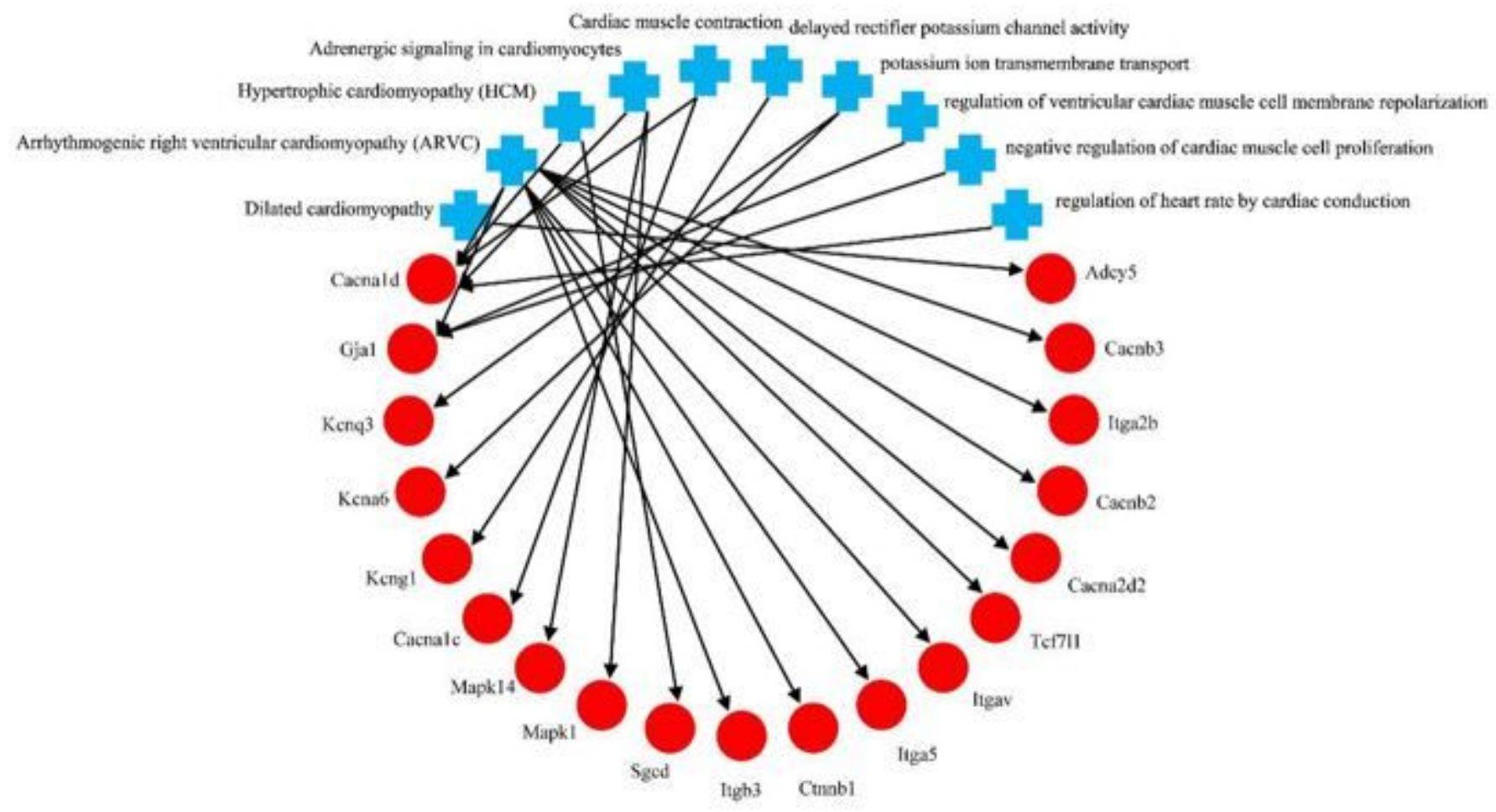

\section{Figure 6}

Interaction of mRNAs with pathophysiological processes associated with RA. Red symbols - mRNA; blue symbols- cardiac pathophysiological processes. cytoscape software data. 

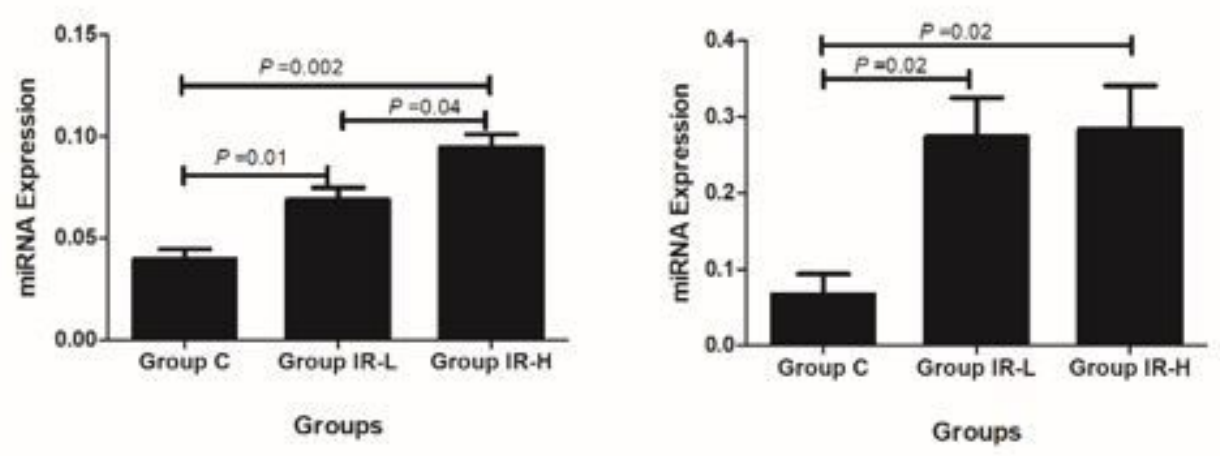

rno-miR-429

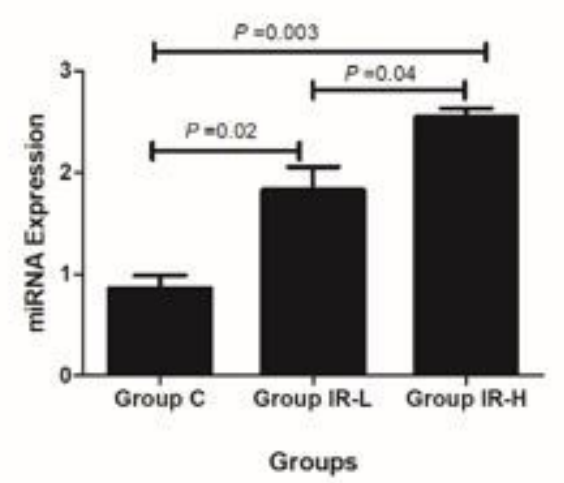

novel_mir19

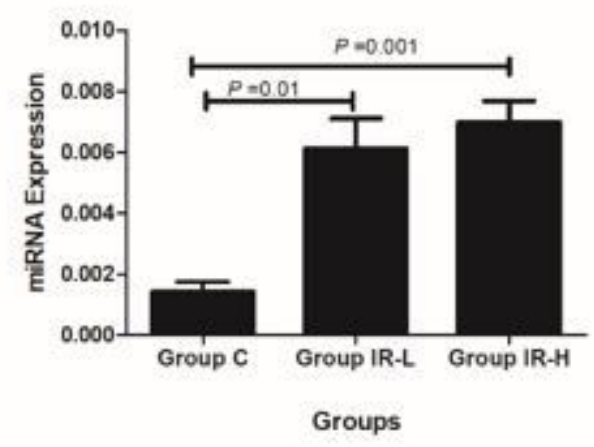

Figure 7

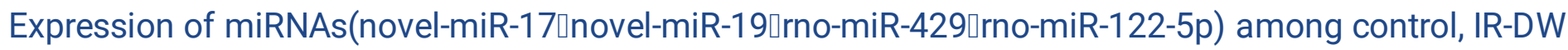
and IR-GW groups. Group names are listed at the bottom. Relative expression was quantified using the comparative CT.

\section{Supplementary Files}

This is a list of supplementary files associated with this preprint. Click to download.

- NC3RsARRIVEGuidelinesChecklistfillable.pdf 